\title{
MEASURING MAGNETIC FIELD OF NEUTRON STAR 4U 0115+63 WITH CYCLOTRON FEATURES
}

\author{
SU YAO* and CHENGMIN ZHANG \\ National Astronomical Observatories, 20A Datun Road \\ Chaoyang District, Beijing 100012, China \\ *yaosu@nao.cas.cn
}

\begin{abstract}
We analyze the $3-80 \mathrm{keV}$ spectrum of X-ray pulsar $4 \mathrm{U} 0115+63$ taken by Rossi X-ray Timing Explorer(RXTE) on April 22nd, 2008. Two absorption features at $\sim 13.5 \mathrm{keV}$ and $\sim 20.5 \mathrm{keV}$ are detected by using cyclotron scattering feature profiles to fit the spectrum, ignoring the inadequate photons of the analyzed data. The fundamental feature is just right between $\sim 11$ and $\sim 16 \mathrm{keV}$. It seems implausible to interprete these absorption lines as cyclotron features because of their inharmonic behavior, even when relativistic effect has been taken into account. But it is possible that the main region where first harmonic line forms is slightly different from the fundamental one.
\end{abstract}

Keywords: Pulsar; magnetic field of neutron star; X-ray observation.

\section{Introduction}

Magnetic field strength is one of the most important physical parameters of neutron star(NS). Since its first detection in $1976,{ }^{1}$ cyclotron lines have been considered to be the most accurate and the only direct way to measure magnetic field near the surface of NSs. Soon after the first detection, cyclotron lines were treated as harmonic lines caused by electron's transition between Landau energy levels, corresponding to a non-relativistic relation $E_{\text {cyc,n }} \sim 11.6 \mathrm{n} B_{12}(1+z)^{-1}$, where $\mathrm{n}$ is the energy level, $B_{12}$ is magnetic field in unit of $10^{12} \mathrm{G}$ and $z$ the gravitational redshift. The subsequent observation $^{2}$ of X-Ray Transient $4 \mathrm{U} 0115+63$ showed the line feature in the spectrum appeared as absorption in $11.5 \mathrm{keV}$ and $23 \mathrm{keV}$. It is suggested that the cyclotron re-emitting processes is equal to scattering and one might expect Cyclotron Resonant Scattering Features(CRSF) as absorption in the spectrum. This is consistent with the observations during the past decades which can be fitted well using absorption features depending on a proper continuum model, though only less than thirty sources, including one ioslated, ${ }^{3}$ have shown credible CRSFs so far. Some of these sources, their spin period, orbital period, fundamental CRSF $E_{\text {cyc, } 1}$ and references related are shown in Table 1.

Though some progresses have been made, ${ }^{14}$ the theoretical model for X-Ray binary pulsar's emission is far from complete. The continuous spectra of accreting sources are usually fitted with the power-law component representing the mixture 
Table 1. X-Ray Pulsars with CRSFs.

\begin{tabular}{cccccc}
\hline Object & $\begin{array}{c}P_{\text {spin }} \\
(\mathrm{s})\end{array}$ & $\begin{array}{c}P_{\text {orbit }} \\
(\text { day })\end{array}$ & $\begin{array}{c}E_{\text {cyc, } 1} \\
(\mathrm{keV})\end{array}$ & $\begin{array}{c}B(1+z)^{-1} \\
\left(10^{12} \mathrm{G}\right)\end{array}$ & Reference \\
\hline 4U 0115+63 & 3.6 & 24.3 & 11.5 & 1 & Ref. 2 \\
4U 0352+309 & 837 & 250 & 28.6 & 2.5 & Ref. 4 \\
4U 1538-52 & 529 & 3.73 & 20.7 & 1.8 & Ref. 4 \\
4U 1626-67 & 7.67 & 0.0298 & 39.3 & 3.4 & Ref. 4 \\
4U 1907+09 & 439 & 8.37 & 18.3 & 1.6 & Ref. 4 \\
Vela X-1 & 283 & 8.96 & 23.3 & 2 & Ref. 5 \\
Cen X-3 & 4.8 & 2.09 & 30.4 & 2.6 & Ref. 4 \\
V0332+53 & 4.37 & 34.25 & 25.2 & 2.2 & Ref. 6 \\
Cep X-4 & 66.3 & 20.8 & 30.7 & 2.6 & Ref. 7 \\
Her X-1 & 1.24 & 1.7 & 34.7 & 3 & Ref. 8 \\
A0535+26 & 103 & 111 & 48.5 & 4.2 & Ref. 9 \\
GX 301-2 & 690 & 41.5 & 42.2 & 3.6 & Ref. 4 \\
J1946+274 & 15.8 & 169.2 & 34.9 & 3 & Ref. 4 \\
MXB 0656-072 & 160.4 & - & 32.8 & 2.8 & Ref. 10 \\
J1409-619 & 500 & - & 21 & 1.8 & Ref. 11 \\
GX 304-1 & 275.5 & 132.5 & 53.7 & 4.6 & Ref. 12 \\
J16493-4348 & 1069 & 6.78 & 33 & 2.8 & Ref. 13 \\
\hline
\end{tabular}

of blackbody, bremsstrahlung radiation, and an exponential cutoff at high energy possibly representing thermal comptonization in accretion column, while for the line features, both Gaussian and Lorentz profiles are employed. See Ref. 15 for details about X-Ray binary pulsar spectrum fitting.

Among the sources with CRSFs, $4 \mathrm{U} 0115+63$ is one of the most studied X-Ray pulsar, and CRSFs were observed up to the fifth. ${ }^{16}$ And the energy of fundamental feature varies from $\sim 11 \mathrm{keV}$ at its maximum luminosity throughout the outburst to $\sim 16 \mathrm{keV}$ at the end of outburst. ${ }^{17}$ In this short work, we analyze the RXTE observation of $4 \mathrm{U} 0115+63$ on April 22nd of 2008 and try to find CRSFs on its spectrum.

\section{Data Analysis and Results}

We concentrated on the observation on April 22nd, 2008 (Observation ID: 9303216-02-01) because of its long observing time, giving totally $\sim 38 \mathrm{ks}$ of exposure time. Standard 2 data of Number 2 Proportional Counter Unit(PCU) in Proportional Counter Array(PCA) on board RXTE is considered since other PCUs were not always working during the observation. We only extracted data from the top layer of PCU for its highest signal to noise ratio. The data reduction employed the HEAsoft version 6.11.1 with standard procedure, and the spectral fitting were performed with XSPEC version 12.7.0.

To describe the continuous spectrum, we use the combination of a powerlaw and high-energy cutoff. ${ }^{2}$ Adding only one CRSF with Lorentz profile ${ }^{15}$

$$
\exp \left[-D \frac{\left(W E / E_{\mathrm{cyc}}\right)^{2}}{\left(E-E_{\mathrm{cyc}}\right)^{2}+W^{2}}\right]
$$




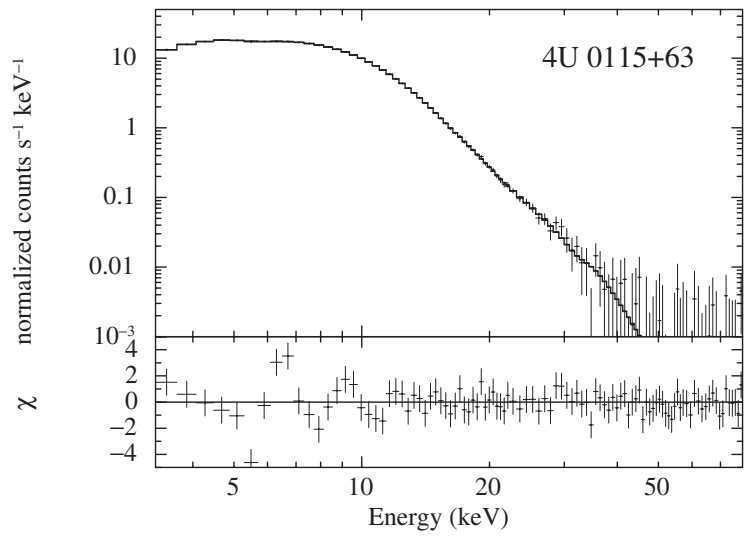

Fig. 1. Top panel: PCA spectrum (cross) and best-fit model (histogram). Bottom panel: Residuals in terms of sigmas with error bars of size one.
Table 2 .

\begin{tabular}{lc}
\hline Model Parameter & Value \\
\hline Photon index & $0.363 \pm 0.013$ \\
$E_{\text {cutoff }}(\mathrm{keV})$ & $13.81 \pm 0.30$ \\
$E_{\text {fold }}(\mathrm{keV})$ & $5.59 \pm 0.53$ \\
$D_{1}$ & $0.94 \pm 0.16$ \\
$E_{\text {cyc, } 1}(\mathrm{keV})$ & $13.63 \pm 0.33$ \\
$W_{1}(\mathrm{keV})$ & $4.80 \pm 0.38$ \\
$D_{2}$ & $0.59 \pm 0.18$ \\
$E_{\text {cyc, } 2}(\mathrm{keV})$ & $20.59 \pm 1.06$ \\
$W_{2}(\mathrm{keV})$ & $5.29 \pm 2.27$ \\
\hline
\end{tabular}

results in $\triangle \chi^{2} \approx 1.39$, showing a line feature at $\sim 14 \mathrm{keV}$. To get a better result, we added another CRSF at $28 \mathrm{keV}$ as first hamonic line which turned into a worse fit $\left(\triangle \chi^{2} \approx 1.42\right)$. Then, with all $D, E_{\text {cyc }}$ and $W$ of two CRSFs left free, the fit is significantly improved to $\triangle \chi^{2} \approx 1.08$ (Fig. 1), which shows the first absorption line appears at $13.6 \mathrm{keV}$ and the second at $20.6 \mathrm{keV}$ (see Table 1 for the best-fit parameters).

These inharmonic features may not be interpreted as cyclotron lines because they scale too far from 1: 2 even when the relativistic effect has been taken into account. The analysis of the data on April 9th, 11th, 13th, 15th and 18th showed that the luminosity of the source has been reducing from April 9th to 22nd. And the insufficiency of the detected photons can affect the analysis. But it is possible, if it is not for the inaccuracy of data, that the inharmonic behavior may be caused when the main region where the first harmonic line forms is slightly different from the fundamental one. Considering the conservation of magnetic moment $B R^{3} \approx$ const, these two regions are $\sim 1 \mathrm{~km}$ apart. This still needs the support from more study about the radiation mechanism and geometry in accretion column.

\section{Acknowledgments}

We are grateful for the helpful discussion with W. Wang and J. Li. This work has been supported by NSFC(No.10773034) and NBRPC(2009CB824800).

\section{References}

1. J. Trümper et al., Astrophys. J. 219 (1978) 105.

2. N. E. White, J. H. Swank and S. S. Holt, Astrophys. J. 270 (1983) 711.

3. G. F. Bignami, P. A. Caraveo, A. De Luca and S. Mereghetti, Nature 346 (2003) 250.

4. W. Coburn et al., Astrophys. J. 580 (2002) 394.

5. I. Kreykenbohm et al., Astronomy \&5 Astrophysics 395 (2002) 129. 
6. K. Pottschmidt et al., Astrophys. J. 634 (2005) 97.

7. V. A. McBride et al., Astronomy \& Astrophysics 470 (2007) 1065.

8. T. Mihara et al., Nature 346 (1990) 250.

9. C. A. Wilson and M. H. Finger, The Astronomer's Telegram, 605 (2005) 1.

10. V. A. McBride et al., Astronomy \&6 Astrophysics 451 (2006) 267.

11. M. Orlandini, V. Sguera, N. Masetti and F. Frontera, ArXiv e-prints (2010) 1012.1218.

12. T. Yamamoto et al., ArXiv e-prints (2011) 1102.4232.

13. A. D'Aì et al., Astronomy \&S Astrophysics 532 (2011) A73.

14. P. A. Becker and M. T. Wolff, Astrophys. J. 654 (2007) 435.

15. K. Makishima, T. Mihara, F. Nagase and Y. Tanaka, Astrophys. J. 525 (1999) 978.

16. C. Ferrigno et al., Astronomy EAAstrophysics 498 (2009) 825.

17. M. Nakajima, T. Mihara, K. Makishima and H. Niko, Astrophys. J. 646 (2006) 1125. 